\title{
A Comprehensive Analysis of Jet Quenching via a Hybrid Strong/Weak Coupling Model for Jet-Medium Interactions
}

\author{
Jorge Casalderrey-Solana ${ }^{\mathrm{a}}$, Doga Can Gulhan ${ }^{\mathrm{b}}$, José Guilherme Milhano ${ }^{\mathrm{c}, \mathrm{d}}$, \\ Daniel Pablos ${ }^{\mathrm{a}}$, Krishna Rajagopal ${ }^{\mathrm{b}}$ \\ ${ }^{a}$ Departament d'Estructura i Constituents de la Matèria and Institut de Ciències del Cosmos (ICCUB), Universitat de Barcelona, \\ Martí i Franquès 1, 08028 Barcelona, Spain \\ ${ }^{b}$ Laboratory for Nuclear Science and Department of Physics, Massachusetts Institute of Technology, Cambridge, MA 02139, USA \\ ${ }^{c}$ CENTRA, Instituto Superior Técnico, Universidade de Lisboa, Av. Rovisco Pais, P-1049-001 Lisboa, Portugal \\ ${ }^{d}$ Physics Department, Theory Unit, CERN, CH-1211 Genève 23, Switzerland
}

\begin{abstract}
Within a hybrid strong/weak coupling model for jets in strongly coupled plasma, we explore jet modifications in ultrarelativistic heavy ion collisions. Our approach merges the perturbative dynamics of hard jet evolution with the strongly coupled dynamics which dominates the soft exchanges between the fast partons in the jet shower and the strongly coupled plasma itself. We implement this approach in a Monte Carlo, which supplements the DGLAP shower with the energy loss dynamics as dictated by holographic computations, up to a single free parameter that we fit to data. We then augment the model by incorporating the transverse momentum picked up by each parton in the shower as it propagates through the medium, at the expense of adding a second free parameter. We use this model to discuss the influence of the transverse broadening of the partons in a jet on intra-jet observables. In addition, we explore the sensitivity of such observables to the back-reaction of the plasma to the passage of the jet.
\end{abstract}

Keywords:

\section{Introduction}

Jet quenching phenomena in heavy ion collisions involve processes that take place at a wide range of scales. The production and initial evolution of jets at high virtualities are under good theoretical control through perturbative QCD. However, the interaction of the jet with the plasma at a temperature somewhat above the transition temperature $T_{c} \sim \Lambda_{Q C D}$ makes the application of current perturbative techniques less appropriate. Indeed, from the fact that the collective properties of the plasma at this range of temperatures are better described as a strongly coupled system than as a weakly coupled gas of quarks and gluons, it is then reasonable to expect non-perturbative physics to play an important role in the jet/QGP interplay. The hybrid strong/weak coupling model presented in [1] takes into account these two facts by applying holographic calculations for the description of this dialogue, while relying on the DGLAP equations perturbatively for the very energetic parton branching.

At the moment there is no complete theoretical framework which can describe both strong and weak coupling processes at different scales in a consistent way. This is why our model is to be considered as 

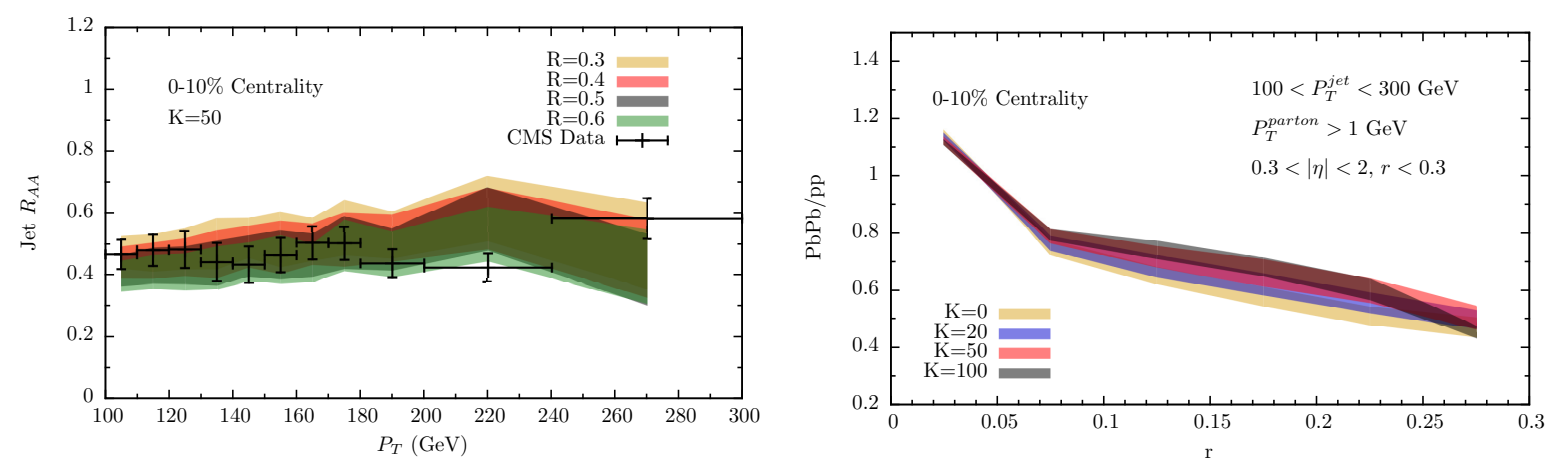

Fig. 1. Left: Jet $R_{A A}$ for $K=50$ for different jet radius $R$. The parameter $\kappa_{S C}$ has been fitted to describe data at $R=0.3$ as measured in [5]. Right: Jet shapes ratio for $R=0.3$ jets for different values of $K$ as compared to experimental data from [6].

a phenomenological approach which benefits from the big separation of scales, from the virtuality to the temperature, to interleave the most relevant physical processes at each scale. Even though it is a simple prescription, the model has proven to be a powerful tool in its confrontation against available data for several jet observables [1, 2, 3], and in generating a broad range of concrete predictions for LHC run II [2]. In these proceedings we show results for the inclusion of parton broadening due to the presence of a thermal bath, and how it is reflected in some of the jet observables measured in experiments. The effect of medium response to the deposition of energy by the jet will also be discussed.

\section{A Hybrid Model}

The hybrid description in our model of jet quenching starts with two main elements. High virtuality splittings occur during a time scale that the medium scale, the temperature, cannot resolve. They are effectively decoupled from the effects of the presence of a colour bath, and are to be treated as if they happened in vacuum, this is through the perturbative DGLAP equations. Between the splittings, and during a time corresponding to the formation time $\tau=2 E / Q^{2}$, the partons interact with the QGP non-perturbatively. This interaction is modelled using insights from the gauge/gravity correspondence by applying the differential energy loss rate found in [4]

$$
\frac{1}{E_{\text {in }}} \frac{d E}{d x}=-\frac{4 x^{2}}{\pi x_{\text {stop }}^{2} \sqrt{x_{\text {stop }}^{2}-x^{2}}} \quad x_{\text {stop }}=\frac{1}{2 \kappa_{S C}} \frac{E_{\text {in }}^{1 / 3}}{T^{4 / 3}},
$$

with $x_{\text {stop }}$ being the maximum length a parton can travel in the plasma before thermalizing. We consider the number $\kappa_{S C}$ to be our single fitting parameter. More details on the physics of the strongly coupled energy loss, as well as further explanations on the Monte Carlo implementation can be found in [1].

In these proceeding we improve our analysis in $[1,2]$ by incorporating two important physics processes, namely the parton broadening and medium response to deposition of energy, which were absent in those works. The first of them has its origin in the Brownian motion a probe experiences in a thermal bath induced by random forces, and its effects consist in partons experiencing transverse kicks (with respect to the fluid frame) with a random direction in this transverse plane and with a strength determined by a Gaussian distribution whose width is $\Delta Q^{2}=\hat{q} d t_{F}$, with $\hat{q}=K T^{3}$ being the transferred transverse momentum squared per unit length. Here, $K$ is a second free parameter which we will leave unconstrained in this first exploratory study.

Medium response is nothing but the overall conservation of momentum. Lost energy from the partons is absorbed into the plasma and transported away at large angles in the form of long wavelength hydrodynamic excitations. We estimate this back-reaction effect on the QGP by assuming small perturbations on top of a Bjorken flow. We consider that the fluctuations are narrow in rapidity around the parton direction. Using 

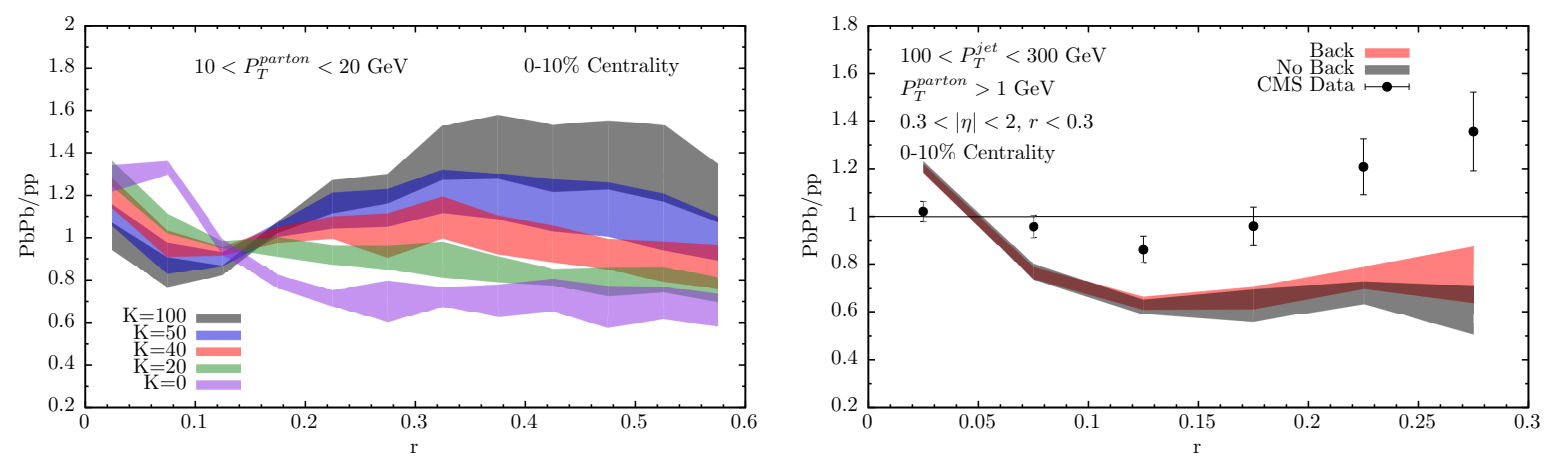

Fig. 2. Left: Jet shapes ratio considering tracks only within the range $10<p_{T}<20 \mathrm{GeV}$ for different values of $K$. Right: Comparison of jet shapes ratio with and without back-reaction effects. Data points taken from [6].

the Cooper-Frye formula, the one body distribution for the extra emission of thermal particles off a boosted, heated up fluid cell reads as

$$
E \frac{d N}{d^{3} p}=\frac{1}{32 \pi} \frac{m_{T}}{T^{5}} \cosh \left(y-y_{j}\right) e^{-\frac{m_{T}}{T} \cosh \left(y-y_{j}\right)}\left[p_{T} \Delta P_{T} \cos \left(\phi-\phi_{j}\right)+\frac{1}{3} m_{T} \Delta M_{T} \cosh \left(y-y_{j}\right)\right],
$$

where $\Delta P_{T}$ and $\Delta M_{T}$ are the lost transverse momentum and mass by the jet respectively, with $y_{j}$ its rapidity and $\phi_{j}$ its azimuthal angle. One can see that negative contributions are present at large azimuthal separation; these diminish the background yield for that region in space. Event by event, we completely constrain the distribution of extra particles via four momentum conservation by making use of a Metropolis algorithm.

\section{Results}

We explore first the inclusion of broadening in different jet observables. We vary $K$ in the range $K \in$ $[0,100]$. To set the scale, the value of $\hat{q}$ extracted by the jet collaboration in [7] approximately corresponds to $K=5$, while the strong coupling estimate of the broadening parameter in [8] yields $K=24$ (for $\lambda=10$ ). Since transverse kicks lead to the transport of soft partons out of the jet cone, the inclusion of broadening contributes to energy loss. For this reason we refit our $\kappa_{S C}$ parameter, finding that even for the highest value of $K$ explored the $\kappa_{S C}$ parameter has to be reduced less than $10 \%$.

In the left panel of Fig. 1 we show jet $R_{A A}$ for different jet radius $R$ with $K=50$. One can see that there is slightly more quenching with increasingly wider radius. The reason why this occurs is independent on the value of $K$; the larger the jet radius, the larger the number of partons in the jet that propagate simultaneously on the plasma, which implies a larger number of sources of energy loss. The small readjustment of $\kappa_{S C}$ when including broadening is saying that little energy is lost due to broadening, meaning that it is no surprise that little energy is recovered by going to wider angles when $K \neq 0$.

We have also studied the effect of broadening on jet shapes. In the absence of broadening $K=0$, our model is unable to reproduced the qualitative behaviour of this observable measured by the CMS collaboration [6]. This is a consequence of the strong quenching in medium: broad jet structures lose more energy since they also possess more sources for energy loss. As a result, in-medium jets in our model are narrower that in vacuum. According to expectations, the inclusion of broadening leads to a widening of the jet shape. However, similar to jet suppression, this effect is very small, even for extreme values of the parameter $K$, insufficient to reconcile our computations with the experimentally observed trend. The small effect of the transverse kicks is a consequence of the small value of $\mathrm{R}$ used to reconstruct the jet. As we have checked, for these narrow jets most of the soft fragments that populate the jet cone are produced late, outside of the medium; soft fragments produced early are strongly quenched and do not contribute to the jet shapes. The overall effect of broadening is, therefore, to change the direction of the jet axis without significantly changing the distribution of fragments inside the jet cone. 
This reasoning, and the motivation to find an observable sensitive to broadening, brings us to the study of a special type of jet shapes, where the track $p_{T}$ range is chosen in such a way that the aforementioned caveats can be dismissed. In the left panel of Fig. 2 we show such a plot for different values of $K$. Even though the jets entering this analysis are reconstructed with $R=0.3$, we extend $r$ up to twice the jet cone so that we see where these semi hard tracks in the range $10<p_{T}<20 \mathrm{GeV}$ end up being as a consequence of the transverse kicks. This observable shows a significant separation for different values of $K$, a fact that makes it a good candidate to quantify the effect that broadening has in experiments.

Since very energetic vacuum jets have only around the $2 \%$ of their energy at the periphery, it is reasonable to expect that little amounts of extra energy falling in such bins in the heavy ion events could have important effects on the ratio of $\mathrm{PbPb}$ vs pp. A natural process capable of producing this effect is the response of the medium to the energy and momentum deposited by the jet constituents, which makes the QGP liquid locally a little hotter and faster. One of the observable consequences can be seen in the right panel of Fig. 2, where we have calculated the jet shapes ratio with and without back-reaction for $K=0$. By estimating the back-reaction effect we observe an important enhancement on the tail of the curve, which although being insufficient to account for the measured shape in experiments, it shows that the inclusion of medium response plays a crucial role in understanding the qualitative behaviour in data. These ideas have also been discussed in [9], arriving to similar conclusions.

\section{Conclusions}

In these proceedings we have presented some results for our hybrid model with the addition of the effects of intra-jet broadening and medium back-reaction. Within our implementation of strongly coupled energy loss, adding broadening does not result in sizeable effects for some observables such as $R_{A A}$ or jet shapes. Nevertheless, we have introduced a new observable, a special version of jet shapes shown in the left panel of Fig. 2, which is able to discriminate among different values of the dimensionless parameter $K$ for the same amount of quenching $\left(R_{A A}\right.$ value).

We have also found that back-reaction plays an important role in the understanding of jet shapes. The wake generated by the jet passage carries the lost energy away at large angles in the form of soft particles, some of which still remain in the jet cone contributing to the observed enhancement of the jet shapes ratio described in data. Our study is only based on a small perturbation analysis of the fluid motion induced by the jet; going beyond small perturbations may increase the importance of collective response on this observable.

Acknowledgements JCS is a University Research Fellow of the Royal Society. JCS was also supported by a Ramón y Cajal fellowship. The work of JCS and DP was supported by the Marie Curie Career Integration Grant FP7-PEOPLE-2012-GIG-333786, by grants FPA2013-46570 and FPA2013-40360-ERC and MDM2014-0369 of ICCUB (Unidad de Excelencia 'María de Maeztu') from the Spanish MINECO, by grant 2014-SGR-104 from the Generalitat de Catalunya and by the Consolider CPAN project. The work of DCG and KR was supported by the U.S. Department of Energy under Contract Numbers DE-SC0011088 and DE-SC0011090, respectively. The work of JGM was supported by Fundação para a Ciência e a Tecnologia (Portugal) under project CERN/FP/123596/2011 and contract 'Investigador FCT - Development Grant'.

\section{References}

[1] J. Casalderrey-Solana, D. C. Gulhan, J. G. Milhano, D. Pablos, K. Rajagopal, A Hybrid Strong/Weak Coupling Approach to Jet Quenching, JHEP 10 (2014) 19. arXiv:1405.3864, doi:10.1007/JHEP10(2014)019.

[2] J. Casalderrey-Solana, D. C. Gulhan, J. G. Milhano, D. Pablos, K. Rajagopal, Predictions for Boson-Jet Observables and Fragmentation Function Ratios from a Hybrid Strong/Weak Coupling Model for Jet Quenching arXiv:1508.00815.

[3] J. Casalderrey-Solana, D. C. Gulhan, J. G. Milhano, D. Pablos, K. Rajagopal, Boson-Jet Correlations in a Hybrid Strong/Weak Coupling Model for Jet Quenching in Heavy Ion Collisions, 2015. arXiv:1511.08174.

[4] P. M. Chesler, K. Rajagopal, Phys.Rev. D90 (2014) 025033. arXiv:1402.6756.

[5] G. Aad, et al., Measurements of the Nuclear Modification Factor for Jets in $\mathrm{Pb}+\mathrm{Pb}$ Collisions at $\sqrt{s_{\mathrm{NN}}}=2.76$ TeV with the ATLAS Detector, Phys. Rev. Lett. 114 (7) (2015) 072302. arXiv:1411.2357, doi:10.1103/PhysRevLett.114.072302.

[6] S. Chatrchyan, et al., Modification of jet shapes in PbPb collisions at $\sqrt{s_{N N}}=2.76 \mathrm{TeV}$, Phys. Lett. B730 (2014) $243-263$. arXiv:1310.0878, doi:10.1016/j.physletb.2014.01.042. 
[7] K. M. Burke, et al., Extracting the jet transport coefficient from jet quenching in high-energy heavy-ion collisions, Phys. Rev. C90 (1) (2014) 014909. arXiv:1312.5003, doi:10.1103/PhysRevC.90.014909.

[8] H. Liu, K. Rajagopal, U. A. Wiedemann, Calculating the jet quenching parameter from AdS/CFT, Phys. Rev. Lett. 97 (2006) 182301. arXiv:hep-ph/0605178, doi:10.1103/PhysRevLett.97.182301.

[9] Y. He, T. Luo, X.-N. Wang, Y. Zhu, Linear Boltzmann Transport for Jet Propagation in the Quark-Gluon Plasma: Elastic Processes and Medium Recoil, Phys. Rev. C91 (2015) 054908. arXiv:1503.03313, doi:10.1103/PhysRevC.91.054908.

[10] P. Arnold, D. Vaman, JHEP 1010 (2010) 099. arXiv:1008.4023. 